Published in: Journal of Alternative and Complementary Medicine (1999) Vol 5, No. 4, pp. 383389.

\title{
Therapeutic Benefits of Qigong Exercises in Combination with Drugs
}

\author{
By \\ Kenneth M. Sancier, Ph.D. \\ Founder, Qigong Institute \\ 561 Berkeley Avenue \\ Menlo Park, CA 94024
}

\begin{abstract}
The present paper reviews (count number) clinical studies from the Qigong Bibliographic Database, developed by the Qigong Institute, East West Academy of Healing Arts, a non-profit organization. This on-line database was started in 1994 and holds approximately 1,000 references going back to 1986, covering medical applications, scientific and experimental studies on Qigong from China, USA and Europe. Records in English have been compiled from International Qigong conferences and seminars, scientific journals, magazines, dissertations, Medline and other databases. The therapeutic role of qigong exercises combined with drugs is reported for three medical conditions that require drug therapy for health maintenance: hypertension, respiratory disease, and cancer. In these studies, drugs were administered to all patients who were divided into two groups, a group that practiced qigong exercises and a control group that did not.

Taken together, these studies suggest that practicing qigong exercises may favorably affect many functions of the body, permit reduction of the dosage of drugs required for health maintenance, and provide greater health benefits than use of drug therapy alone. The reported studies do not necessarily measure up to the strict protocols required for randomized controlled clinical trials. For hypertensive patients, combining qigong practice with drug therapy for hypertensive patients resulted in reduced incidence of stroke and mortality and reduced dosage of drugs required for blood pressure maintenance. For asthma patients, the combination therapy permitted reduction in drug dosage, the need for sick leave, duration of hospitalization, and costs of therapy. For cancer patients, the combination therapy reduced the side effects of cancer therapy. Also reported is a study showing that the practice of qigong helps to rehabilitate drug addicts.
\end{abstract}




\section{INTRODUCTION}

Qigong is an ancient form of health maintenance dating back thousands of years and is believed to be the basis of traditional Chinese medicine (Cohen, 1997). The word qigong (pronounced chee gong) contains two concepts: qi, the vital energy of the body, and gong, the training or cultivation of the qi. Qigong practice consists of a series of exercises including breathing, meditation, and rhythmical movements that can be learned by almost anyone of any age or physical condition. Qigong claims to foster health and healing by promoting a smooth flow of qi throughout the body so that the body can heal itself. The practice of qigong can be divided into two main categories: medical and martial. This article is devoted entirely to medical qigong, and the main emphasis will be on self-practice of qigong exercises in contrast to qigong healing by a qigong master.

It is important to distinguish qigong from traditional Chinese medicine (TCM). TCM diagnosis includes questioning of the patient and sophisticated examination of the tongue, eyes, and the pulses at the radial artery of the wrist; treatments include acupuncture, moxibustion, herbal remedies, massage, and nutrition. For qigong, diagnosis includes those of TCM together with sensing of the body field for blocks to the flow of qi; treatments include qigong exercises and in serious cases treatment by qigong therapies. In China it is estimated that 70 million Chinese practice qigong daily (McGee and Chow, 1994). There are many qigong clinics, and in some hospitals qigong is integrated with TCM and with conventional western biomedicine. These distinctions are discussed more fully in a recent article (Cassidy, 1998). Qigong is said to have several thousand forms, with some designed to benefit certain diseases while most others have general health benefits.

Several complementary medical therapies with some similarities to qigong are practiced in hospitals in the west and are paid for by insurance. Yoga is prescribed as part of the therapy for heart disease (Ornish, 1990), and Dean Ornish's cardiac health program is covered by 40 major insurance companies and administered in 11 hospitals (Ornish, 1998). Therapeutic Touch has been taught to thousands of nurses and is practiced in many hospitals (Krieger, 1997); eighty North American hospitals include Therapeutic Touch (Field, 1995). T'ai Chi Ch'uan, which derives from Chinese martial arts and which is considered a form of qigong, was part of a study on the effects of exercise to prevent falls of elderly patients (Province, et al., 1995). Also, John KabatZinn's Mindfulness-Based Stress Reduction Therapy is offered in more than 100 US hospitals or clinics (Kabat-Zinn, 1991).

\section{CLINICAL STUDIES EXAMINING THE BENEFITS OF QIGONG PRACTICE}

\section{Hypertension}

Beginning about 1971, researchers at the Shanghai Institute of Hypertension conducted a series of retrospective and prospective research projects following the progress of hypertensive patients. These studies are discussed and reviewed in an accompanying article (Mayer, 1999). The following brief descriptions of the studies are provided for bibliographic reference: 
In Xu and Qiang Wang (1991a), the experimental group practiced qigong exercises, "Bi Bing Yang Sheng Gong," for 30 minutes twice a day and the control group did not. This style of qigong is claimed to be especially valuable for therapeutic purposes and delaying senility, and consists of a combination of sitting and standing meditation and gentle physical movements that emphasize a calm mind, relaxed body, and regular breathing. To control the hypertension, all patients were given hypotensive drugs, namely, reserpine, dihydrazine and dihdrochlorothiazide, with dosage adjusted to need.

Three retrospective studies from the same clinic include:

- A 20-year retrospective study of 204 patients who were randomly assigned to two groups (Kuang et al., 1991a\&b);

- A 30 year retrospective follow-up of 242 hypertensive patients (Wang et al, 1993); - An 18-22 year study of 536 patients (Wang, 1997)

It is claimed from these studies that combining qigong practice with drug therapy for hypertensive patients resulted in reduced incidence of stroke and mortality and the ability to reduce dosage of drugs required for blood pressure maintenance.

\section{Sex hormone levels}

As people age, sex hormone levels change, specifically, testosterone levels fall in men and increase in women, while estrogen levels rise in men and fall in women, and abnormal changes in these levels have been linked with hypertension. Two groups (Ye, 1990; Kuang et al., 1991a) studied the influence of qigong exercises may have in normalizing these values and both empirically demonstrated potential benefits. They reported similar favorable changes in plasma sex hormone levels--numbers approaching those of healthy individuals--in both males and females practicing qigong.

\section{Respiratory Disease}

The following two studies suggest that qigong exercise may play a role in improving the condition of patients with respiratory disease. The results also suggest that combination therapy of qigong and drugs yield better results than drug therapy alone for reducing the symptoms of respiratory disease, improving the immune and the cardiovascular systems, and increasing the general conditions of the patients.

\section{Bronchial asthma}

Bronchial asthma is a chronic respiratory disorder with acute deterioration. Therapy should show improvement in one or more of the following: acute respiratory distress relief, reduction in chronicity, medication, hospitalization or treatment costs. The following reports shows that according to these criteria, qigong practice has a beneficial effect in the treatment of asthma.

Reuther and Aldridge (1998) demonstrated that over a period of 52 weeks, qigong exercise did indeed help reduce the number of different medications as well as the number of applications of the different medications, and decreased the variability of peak respiration flow. Patients who did not practice qigong regularly showed less 
improvement in symptoms, less change in medication required, and greater variability in peak respiration flow. Figure 1 shows the results of the one year study.

Based on the patients' medical histories, Reuther and Aldridge estimated that there would be substantial cost savings from the practice of qigong. After one year there would be significant decreases in sick leave days, hospitalization days, emergency consultation, and administration of antibiotics for bronchial infection.

\section{Chronic respiratory diseases}

Li, Liu, and Zhou (1998) conducted a comparative study over 3 months, examining the effects of drugs and qigong exercise on patients with chronic respiratory diseases (e.g. chronic bronchitis, asthma, and pulmonary emphysema). Greater benefits were observed when drugs were administered in combination with qigong practice (lower average breathing rates and heart rates) than when taken alone.

The results from these two studies suggest that combination therapy of qigong exercises and drugs is superior to drug therapy alone for reducing the symptoms of respiratory disease, improving the immune and the cardiovascular systems, and increasing the general conditions of the patients.

\section{Cancer}

Examination of the effects of qigong exercise combined with drug therapy suggests a relationship with an extended tumor-free survival of the patients, decreased size and hardness of tumors, reduced side effects of chemotherapy and radiation therapy, improved immune function, and improved quality of life. These results offer promise that the practice of qigong could effectively complement Western medical therapies. Several studies on the comparative effects of qigong exercise alone, drugs alone, and both in combination, on cancer patients are discussed below. In all studies, drugs were administered to control cancer or the side effects of cancer therapy.

\section{Case Studies}

- Xu Hefen and colleagues studied changes in the immunological functions of patients who practice qigong (Xu and Qi, 1988, 1989). They compared the effects of practicing or not practicing qigong for patients with malignant tumors versus healthy people by assessing their immune activity and function (T-lymphocyte count, serum immuno-protein production (IgG, $\operatorname{IgA}$, and $\operatorname{IgM}$ ) leukocyte adnerence inhibition (LAI) tests, and active E rosette formation (Ea) tests). The values for T-lymphocytes were observed to be higher for subjects who practiced qigong exercise than those that did not, whether healthy subjects or patients with tumors (see Table 4). In accordance with this finding, the values for IgG, LAI, and Ea were also enhanced. The researchers state that, from this data, qigong exercise may enhance human immunological functions, possibly resulting in greater resistance and more rapid recovery from diseases such as malignant tumors. 
- Sun and Zhao conducted a clinical study of qigong exercise as a therapeutic aid for patients diagnosed with advanced cancer (Sun and Zhao, 1988), showing improvements (four to nine times greater than the control group) in strength, appetite, being free of diarrhea, and weight gain of $3 \mathrm{~kg}$. The phagocytic rate, a measure of the immune function, increased in the group practicing qigong but decreased in the control group.

- Wang and colleagues advised their cancer patients to persist in long-term qigong exercises during routine treatments such as chemotherapy, radiotherapy and surgery (Wang, Wang. Shao and Li, 1993). Their findings revealed that the general health of the qigong group improved with most of them having a stable or slightly declining white blood count, and chemotherapy was suspended among $92 \%$ of this group (suggesting cancer had been controlled in these patients). The general health of the control group deteriorated with a decline in white blood cell count, and chemotherapy was suspended for only $61 \%$.

- In another study, patients with gastrointestinal tumors were divided into a group practicing qigong and a control group (Peng et al., 1989). Patients in both groups were treated appropriately with surgery, chemotherapy, radiotherapy and Chinese herbal medicine. Among those practicing qigong, the functions of the stomach and intestines improved as evidenced by an increase over the control group in the patients' appetite (eg., caloric intake rose from 870 to $1560 \mathrm{cal} /$ day), white blood cell count (to normal), and survival rates ( $80 \%$ vs. $65 \%$ after three years, and $45 \%$ vs. $30 \%$ after five years, respectively).

- In 1993, Fu studied the effects of qigong exercises on patients with gastric carcinoma who lost the chance for an operation because of their advanced age, weakness or because their disease was in an advanced stage at first presentation. Patients were divided into a group practicing qigong and a control group, and both groups received the anti-cancer Chinese herbal drug "Weiliuping." The results showed that qigong exercise may help to promote health since the group practicing qigong improved more than the control group in relief of symptoms and in the index of immunity.

- Cong and colleagues reported the effects of qigong exercises on patients suffering from medium-late staged esophageal cancer by supplementing their combination therapy of Chinese herbs (Tianxian capsules), radiotherapy and/or chemotherapy (Cong et al., 1996) with qigong. Results showed that practicing qigong most likely increased the remittive effect, decreased side effects, and improved the quality of life.

\section{Drug Addiction}

Ou and $\mathrm{Li}$ (1998) studied the benefits of qigong exercises for eliminating narcotic drugs from the body of drug addicts. Not only was the practice of qigong shown to help facilitate clearance of drugs from the system more quickly and thoroughly than the control, but it was also associated with improved sleep, mental condition (e.g. stress and craving), and relief of withdrawal symptoms (e.g. tearing, sweating, salivating, yawning, weakness, chills and muscular pain). 


\section{Discussion}

\section{Mechanism of qigong's action}

If qigong exercise is beneficial, one may speculate that an increase in blood circulation may help to explain the effect of qigong exercises on many different functions of the body. Increased blood circulation enhances delivery of oxygen and nutrients to cells of the body and increases the efficient removal of waste products from the cells. These processes may help nourish diseased or stressed tissue, increasing the efficiency of body functions including immune response, finally enabling the body to heal itself. According to qigong theory, qigong exercise helps remove blocks to the flow of qi and thereby helps balance the flow of qi in the body. One main tenet of qigong and TCM is that "the qi leads the blood," which is to say that when qi flows smoothly and easily, blood will also flow smoothly and easily. One may paraphrase this concept: qi is the potential energy driving the flow of blood, and the practice of qigong is one means to cultivate the qi.

\section{Qigong and blood circulation.}

Several research studies support the proposal that qigong exercise increases blood circulation. Wang and colleagues demonstrated that qigong exercise increased the functions of the heart and capillary blood circulation in nailfolds (Wang et al., 1995). They quote two further studies, published only in Chinese, reporting that the practice of qigong improves the function of the brain and the autonomic nervous system and reduces the disturbance of blood rheology. In another study qigong exercise was shown by rheoencephalography to increase blood flow to the brain for 158 subjects with cerebral arteriosclerosis who practiced qigong for 1 to 6 months (Liu, He and Xie, 1993). Improvements were noted in symptoms such as memory, dizziness, insomnia, tinnitus, numbness of limbs, and vertigo headache. A decrease in plasma cholesterol was also claimed.

Mo and colleagues performed several studies to determine the effect of qigong exercises on cardiovascular function and altitude sickness. In one study they reported that pilots were randomly assigned to two groups before entering the higher altitudes of the Tibetan plateau: a qigong group of 22 men who practiced qiyuan Qigong exercise for eight weeks and a group of 18 men who practiced calisthenic exercises for eight weeks (Mo et al., 1993). When the men entered the high altitude, abnormal blood pressure and decreased capillary blood circulation of tongue apex and nailfold was reported in both groups. The abnormalities were statistically less in the qigong exercise group than in the calisthenic exercise group $(\mathrm{p}<0.01)$. The temperature at the Laogong point, an acupuncture point at the center of the palm, kept steady in the qigong exercise group but was reduced in the calisthenic exercise group $(\mathrm{p}<0.05)$, suggesting that blood circulation was better in the group practicing qigong.

Thermography has been reported to demonstrate that qigong exercises can increase skin temperature, which implies greater blood circulation in particular areas of the body. Machi examined physiological changes before and during qigong practice of several qigong masters, and claimed that both internal, self-performed qigong practice, 
and also external qigong healing, emitted qi or waiqi transmitted from doctor to patient, affected brain waves, blood pressure heart rate and skin temperature (Machi, 1994).

\section{Augmenting Drug Therapy}

As mentioned above, qigong exercises may enhance blood circulation, thereby increasing the efficiency of delivery of drugs in the bloodstream, as well as removing waste products from the body. Omura claimed that drug uptake was increased by using "qigongized paper" (i.e., paper to which emitted qi was sent) applied to an afflicted area (spastic muscle or arteries in vasoconstriction) of the body (Omura, 1990), reducing or eliminating the pain, and relieving the spastic muscles and the circulatory disturbances. This increase in blood circulation may also explain qigong's apparent effect in accelerating removal of narcotic drugs from the body of addicts, in that increased blood flow might enable narcotic drugs in cells to be carried away in the blood stream.

\section{SUMMARY}

There are many clinical studies on the medical benefits of qigong practices but too few are good scientific studies. More scientific research is needed to validate the promising results already reported. Research is required to separate benefits of therapy by qigong exercises alone, qigong exercises with drugs, and drugs alone. Some clinical studies reported such comparisons, but the protocols used or the reported results are not sufficiently well described to allow for evaluation or replication.

Not all qigong practice is of equal value for all people or for all health problems. Future research investigators should select the appropriate type of qigong practice, even for healthy people. However, the benefits of qigong exercises are sufficiently well established to provide credibility for physicians who wish to recommend qigong practice to selected patients or patient groups. In China, there are many qigong clinics. To integrate the practice of qigong into Western healthcare, it would be valuable to examine how China uses qigong practice, along with RCM and western medicine in its system of healthcare.

\section{Acknowledgment}

The author gratefully acknowledges Ellen Friedlander for her insightful suggestions and editorial assistance. 


\section{References}

Cassidy, CM. Chinese medicine users in the United States Part II: preferred aspects of care. J Altern. Complement. Med 1998;4(2):189-202.

Cohen, K. The Way of Qigong, ìThe Art and Science of Chinese Energy Healingî 1997 Ballantine Books, NY.

Cong J, Zhang Q, Chen J, Yang A. Clinical study on the treatment of medium-late staged esophageal cancer by Chinese-Western medicine and qigong therapy. 3rd World Conf for Acad Exch of Medical Qigong. Beijing, China 1996:132.

Field, FD. 1995, August 20; Columbus Dispatch:1-2.

$\mathrm{Fu}$, J. Treatment of advanced gastric cancer in the aged by the combination of qigong and medicinal herbs. 2nd World Conf for Acad Exch of Medical Qigong. Beijing, China 1993:132.

Huang X. Clinical observation of 204 patients with hypertension treated with qigong. 1st Intl Cong of Qigong, UC Berkeley, Calif, USA 1990: 101.

Kabat-Zinn J, Borysenko J, Nhat T, Kabat Z. "Full Catastrophe Living: Using the Wisdom of Your Body and Mind to Face Stress, Pain and Illnessî 1991 Dell Publishing Company, Inc. New York.

Krieger D. 1997 Therapeutic Touch Inner Workbook, Bear, Santa Fe, NM.

Kuang A, Wang C, Xu D, Qian Y Research on ìanti-agingî effect of qigong. J Traditional Chinese Medicine. 1991;11(2):224-227.

Kuang A, Wang C, Xu D, Qian Y. Research on the anti-aging effect of qigong. J Traditional Chinese Medicine 1991a;1(2):153-158.

Kuang A, Wang C, Xu D, Qian Y. Research on the anti-aging effect of qigong. J Traditional Chinese Medicine 1991b;1(2):224-227.

Lazarou J, Pomeranz BH, Corey PN. Incidence of adverse drug reactions in hospitalized patients, a meta-analysis of prospective studies. JAMA 1998;279(15):1200-1205.

Liu Y, He S, Xie, S. Clinical observation of the treatment of 158 cases of cerebral arteriosclerosis by qigong. 2nd World Conf for Acad Exch of Medical Qigong, Beijing, China 1993:125.

Li Z, Liu F, Zhou R. Observations of the results of drug and qigong therapy for chronic respiratory diseases. 1st World Conf Acad Exch Medical Qigong. Beijing, China. 1988:109. 
Machi Y. Various measurements to qigong masters for analyzing qigong mechanism. J. Mind-Body Science. 1994; (1):65-87 [Abstract in English, article in Japanese].

McGee and Chow. "Miracle Healing from China, Qigong" 1994, Medi Press, Idaho.

Mo F, Wan L, Jia Z, Xu G. Study of prevention of microcirculation disorders of pilots in highlands by qigong. Proceedings, Second World Conf on Academic Exchange of Medical Qigong, Beijing, China.1993:78.

Omura Y. Storing of qi gong energy in various materials and drugs (qigongization): its clinical application for treatment of pain, circulatory disturbance, bacterial or viral infections, heavy metal deposits, and related intractable medical problems by selectively enhancing circulation and drug uptake. Acupunct Electrotherapeutics Res, Intl J. 1990;15(2):137-57.

Ornish, D Healing of Hearts. 1998, March 16; Newsweek:50-56.

Ornish D. 1990 Reversing Heart Disease. Ballatine Books, New York.

$\mathrm{Ou} \mathrm{W}$ and Li M. A preliminary exploration into the mechanism of drug cessation by Pangu qigong. Proceedings, 4nd World Congress on Qigong 1998:138-139.

Peng Z, Chen X, Wen Y, Ni Y, Deng G. Clinical observation of 20 patients with malignant gastrointestinal tumors treated by supplementary qigong. 2nd Intl Conf on Qigong. Xian, China. 1989:167.

Province MA, Hadley E, Hornbrook M, Lipsitz L, Miller J, Mulrow C, Ory M, Sattin R, Tinetti, $\mathrm{M}$ and Wolf $\mathrm{S}$. The effects of exercise on falls in elderly patients. JAMA 1995:273(17):1341-1347.

Reuther I, Aldridge D. Treatment of bronchial asthma with qigong yangsheng-a pilot study. J Altern. Complement. Med 1998;4(2):173-183.

Sancier K M, Hu B. Medical applications of qigong and emitted qi on humans, animals, cell cultures \& plants: review of selected scientific research. Am J Acupuncture 1991;19(4):367-377.

Sancier K. Anti-aging benefits of qigong. Proceedings, 2nd World Congress on Qigong 1997:47.

Sancier KM. Medical applications of qigong. Altern Ther Health Med. 1996a;1(4):40-46.

Sancier KM. Anti-aging benefits of qigong. J International Society of Life Information Science 1996b;14(1):12-21. 
Sancier KM. Qigong and drug therapy is superior to drug therapy alone 3rd World Conf for Acad Exch of Medical Qigong. 1996c Beijing, China:171.

Spiegel D. Psychosocial aspects of breast cancer treatment. Seminars in Oncology 1997;24(1):36-47.

Sun Q, Zhao L. Clinical observations of qigong as a therapeutic aid for advanced cancer patients. Proceedings First World Conf Academic Exchange of Medical Qigong, China. 1988:97-98.

Wang C, Xu D, Qian Y, Kuang A. The beneficial effect of qigong on hypertension incorporated with coronary heart disease. Proceedings, Third International Symposium on Qigong, Shanghai, China 1990:40.

Wang C, Xu D, Qian Y, Medical and health care qigong. J Traditional Chinese Medicine 1991a;11(4):296-301.

Wang S, Wang B, Shao M, Li Z. Clinical study of the routine treatment of cancer coordinated by qigong. 2nd World Conf for Acad Exch of Medical Qigong. Beijing, China 1993:129.

Wang C, Xu D, Qian Y, Shi W. Effects of qigong on preventing stroke and relaxing the multiple cerebro-cardiovascular risk factors - follow up of 242 hypertensive patients for 30 years. 2nd World Conf Academic Exch Medical Qigong, Beijing, 1993:123.

Wang C, Xu D, Qian Y, Shi W, Bao Y, Kuang A. Beneficial effects of qigong on the ventricular function and microcirculation of deficiency in heart-energy hypertensive patients. Chinese J Internal Medicine 1995;1(1):21-23.

Wang C. Personal communication. 1997.

Xu, H, Qi Y. Preliminary study on qigong and immunity. 2nd Intl Conf on Qigong. Xian, China 1989:99.

$\mathrm{Xu}$ D, Wang C. Clinical study of delaying effect on senility by practicing iYang Jing Yi Shen Gongî in hypertensive patients. Proceedings, 5th International Symposium on Qigong, Shanghai, China 1994:109.

Xu H, Wang G, Xue H, Zhang C, Wang J, Qi Y. Observation of T-lymphocytes by ANAE staining in the clinical application of qigong. 1st World Conf for Acad Exch of Medical Qigong. Beijing, China 1988:52.

Ye M. Relationship among erythrocyte superoxide dismustase activity, plasma sexual hormones (T, E2), aging and qigong exercise. Proceedings, 3rd International Symposium on Qigong, Shanghai, China. 1990:28-32. 
Ziegler J The mind, the body and the benefits budget. Business \& Health, February $1997 ; 15(2): 22-28$.

Fig. 1. The effect of qigong practice on total number of medications and peak flow variability of an asthmatic patient. Numbers above columns are the number of medicines (From Reuther and Aldrich [1998]).

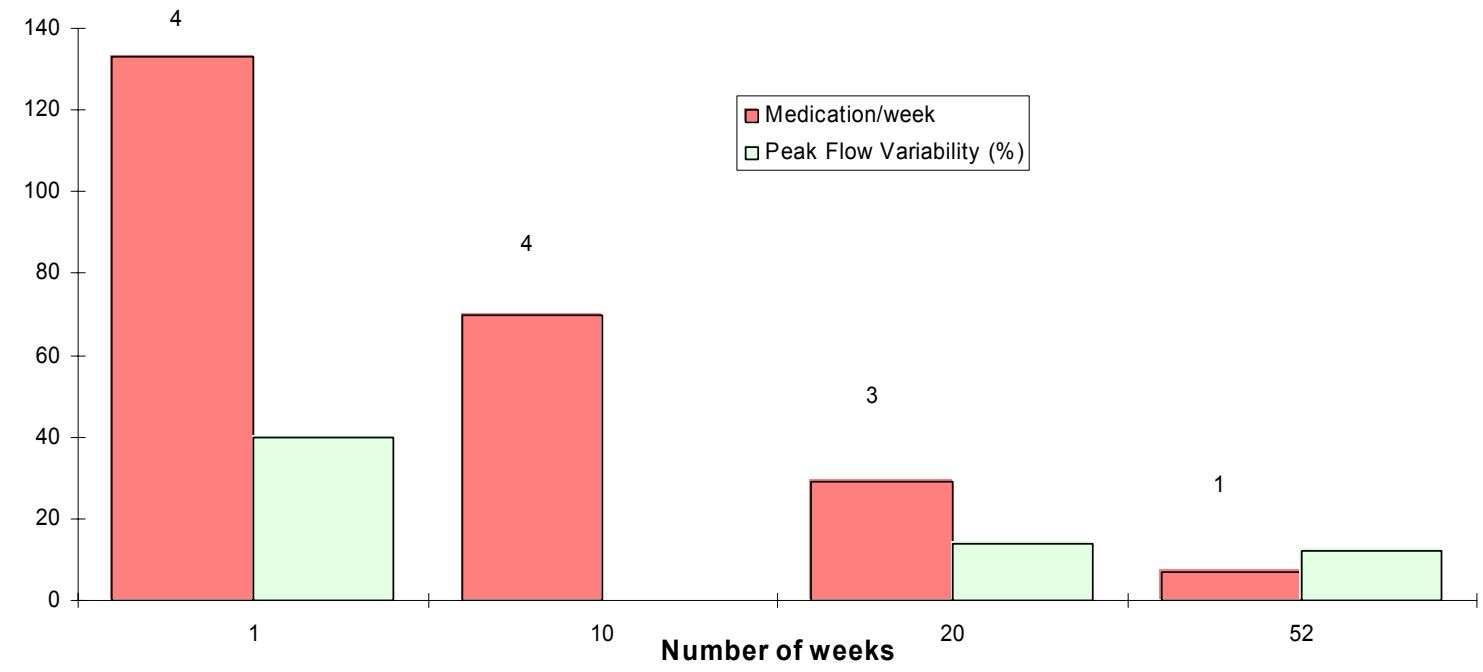

\section{FIGURE CAPTIONS}

Fig. 1. The effect of qigong practice on total number of medications and peak flow variability of respiration of an asthmatic patient. Numbers above columns are the number of medications (from Reuther and Aldridge, 1998). 